\title{
CTLA-4 +49A/G Polymorphism Increases the Susceptibility to Bladder Cancer in Chinese Han Participants: A Case- Control Study
}

\author{
Fei Mao, Xiao-Bing Niu, Shuo Gu, Lu Ji, Bing-Jian Wei, and Heng-Bing Wang \\ Department of Urology, The Affiliated Huaian No.1 People's Hospital of Nanjing Medical University, Huaian, Jiangsu, China \\ Correspondence should be addressed to Heng-Bing Wang; wanghengbing2004@163.com
}

Received 21 November 2019; Revised 1 February 2020; Accepted 5 March 2020; Published 1 December 2020

Academic Editor: Eric A. Singer

Copyright ( 92020 Fei Mao et al. This is an open access article distributed under the Creative Commons Attribution License, which permits unrestricted use, distribution, and reproduction in any medium, provided the original work is properly cited.

\begin{abstract}
Cytotoxic T cell antigen-4 (CTLA-4) is reportedly involved in the development of bladder cancer (BC). This research was designed to address the potential link between the $+49 \mathrm{~A} / \mathrm{G}$ polymorphism in CTLA-4 gene and BC susceptibility. In total, $355 \mathrm{BC}$ cases and 435 match controls from Chinese Han individuals were included eventually. The PCR-RFLR method was utilized to screen for this polymorphism. The $+49 \mathrm{~A} / \mathrm{G}$ polymorphism was shown to increase the risk of BC. Subgroup analyses showed that this polymorphism was linked to an increased susceptibility to $\mathrm{BC}$ among individuals aged $<60$ years, smokers and drinkers. Additionally, this polymorphism significantly correlated with tumor node metastasis and tumor size $(\geq 3 \mathrm{~cm})$. To sum up, this study reveals that the CTLA- $4+49 \mathrm{~A} / \mathrm{G}$ polymorphism could increase the risk of BC in Chinese Han people. Further large cohort studies with enough sample sizes are urgently warranted to verify the findings of this present study.
\end{abstract}

\section{Introduction}

Bladder cancer (BC) is reported to be one of the most common genitourinary tumors [1]. BC is reportedly the ninth most common cancer worldwide, and the incidence rates of $\mathrm{BC}$ in men are the highest in Southern and Western Europe, Western Asia, and North America [2]. BC incidence in women is considerably lower than in men, although difference in incidence between the genders varies among countries [2]. Although genetic predisposition, chronic irritation, and certain environmental conditions have been reported as potential risk factors for BC [3-6], the main risk factor is tobacco smoking [7]. GWAS studies identified susceptibility loci for BC [8-10].

Cytotoxic T cell antigen-4 (CTLA-4), homologous to the $\mathrm{CD} 28$ protein receptor, negatively regulates the $\mathrm{T}$ cell proliferation and activation [11]. By interfering with $\mathrm{T}$ cell activation, CTLA- 4 hampers antitumor-related immune responses and promotes tumor growth [12]. Zhang et al. observed that disrupting the CTLA- 4 expression on $\mathrm{CD}^{+} \mathrm{T}$ cells promoted antitumor immune responses in $\mathrm{BC}$ [13]. Additionally, CTLA-4 inhibition could suppress the growth of BC [14].
Immunotherapy containing anti-CTLA-4 antibodies showed promise as a curative approach for BC [15].

The CTLA-4 gene is shown to locate on chromosome 2q33. Previous studies have suggested a link between CTLA- $4+49$ A/G polymorphism and several types of cancer risk, including hepatocellular carcinoma [16], colorectal cancer [17], and osteosarcoma [18]. Two studies investigating the relationship between $+49 \mathrm{~A} / \mathrm{G}$ polymorphism and BC susceptibility in different populations yielded inconsistent results $[19,20]$. This study was intended to address whether $+49 \mathrm{~A} / \mathrm{G}$ polymorphism in CTLA-4 gene was related to BC susceptibility in this population.

\section{Methods}

2.1. Participants. This research enrolled 355 BC patients eventually. Details regarding the inclusion criteria for the $\mathrm{BC}$ group selection were described in a previous study [21]. During the same period, 435 healthy age- and sex-matched controls receiving a regular health checkup at the same hospital were also recruited. All patients and controls provided 
written informed consent; clinical information was collected using a structured questionnaire. The study was consented by the Ethics Committee of our hospital and was in line with the Declaration of Helsinki.

2.2. Genotyping. By utilization of the QIAamp DNA Blood Mini Kit from QIAGEN, Hilden, Germany, all DNA samples were acquired from EDTA-peripheral blood. The PCR-RFLP method could genotype CTLA-4 +49A/G polymorphism. The PCR primers included $5^{\prime}$-AAGGCTCAGCTGAACC TGGT- $3^{\prime}$ and $5^{\prime}$-CTGCTGAAACAAATGAAACCC- $3^{\prime}$. To confirm the reproducibility of this method, $10 \%$ of the samples were regenotyped, and their consistence was $100 \%$.

2.3. Statistical Analysis. The chi-squared $\left(\chi^{2}\right)$ test was used to analyze categorical variables; the Student's $t$-test was utilized to determine continuous variables. Hardy-Weinberg equilibrium (HWE) was used to test for CTLA $-4+49 \mathrm{~A} / \mathrm{G}$ polymorphisms among healthy individuals. Logistic regression was to evaluate ORs and their 95\% CIs. $P$ value of $<0.05$ indicated statistically significant results. All relevant statistical analyses were performed by the SPSS software package (ver. 22.0; SPSS Inc., Chicago, IL, USA) or the SAS software (version 9.1.3; SAS Institute, Cary, NC, USA).

\section{Results}

3.1. Demographic Data. The demographic data of all participants are detailed in Table 1. Information about tumor grade, tumor size, TNM stage, tumor node metastasis, distant metastasis, and histology is also provided in Table 1 for $\mathrm{BC}$ patients. We found the incidences of sex $(P=0.464)$ or age $(P=0.541)$ did not differ significantly. However, the smoking and drinking rates were higher in BC patients compared with healthy individuals.

3.2. CTLA-4 +49A/G Polymorphism and Risk of BC. The genotype distribution of this polymorphism in healthy individuals was determined by use of the HWE test; no significant enrichment was found. The GG and $A G+G G$ genotypes showed an increased risk for BC (Table 2). These associations still reached significant after adjusting for gender and age. Additionally, the $\mathrm{G}$ allele increased the risk of $\mathrm{BC}(\mathrm{G}$ versus $A$ : $O R, 1.39 ; 95 \% C I, 1.09-1.77 ; P=0.008)$. The subgroup analyses stratified by age, sex, drinking, and smoking are shown in Table 3. Smoking, drinking and age were related to an increased risk of $\mathrm{BC}$, but no significant association was shown in the sex subgroup analysis.

3.3. CTLA-4 +49A/G Polymorphism Relates to Certain Clinicopathological Characteristics of $B C$. We also interpreted the link between this polymorphism and the clinicopathological characteristics of BC patients. We observed that $+49 \mathrm{~A} / \mathrm{G}$ polymorphism had a connection with tumor node metastasis and tumor size (Table 4). However, no potential association between CTLA-4 +49A/G polymorphism and tumor grade, distant metastasis, TNM stage, or histology of BC was indicated.
TABle 1: Patient demographics and risk factors in bladder cancer.

\begin{tabular}{|c|c|c|c|}
\hline Variable & Cases $(n=355$ & $\begin{array}{l}\text { Controls } \\
(n=435)\end{array}$ & $P$ \\
\hline Age (years) & $60.81 \pm 10.57$ & $61.25 \pm 9.73$ & 0.541 \\
\hline Sex & & & 0.464 \\
\hline Male & $303(85.4 \%)$ & $363(83.4 \%)$ & \\
\hline Female & $52(14.6 \%)$ & $72(16.6 \%)$ & \\
\hline Smoking & & & $<0.001$ \\
\hline Yes & $251(70.7 \%)$ & $180(41.4 \%)$ & \\
\hline No & $104(29.3 \%)$ & $255(58.6 \%)$ & \\
\hline Drinking & & & $<0.001$ \\
\hline Yes & $233(65.6 \%)$ & $173(39.8 \%)$ & \\
\hline No & $122(34.4 \%)$ & $262(60.2 \%)$ & \\
\hline \multicolumn{4}{|l|}{ Tumor grade } \\
\hline High $(G 2+G 3)$ & $227(63.9 \%)$ & & \\
\hline Low (G1) & $128(36.1 \%)$ & & \\
\hline \multicolumn{4}{|l|}{ Tumor size $(\mathrm{cm})$} \\
\hline$<3$ & $263(74.1 \%)$ & & \\
\hline$\geq 3$ & $92(25.9 \%)$ & & \\
\hline TNM stage & & & - \\
\hline I & $78(22.0 \%)$ & - & - \\
\hline II & $100(28.2 \%)$ & & \\
\hline III & $104(29.3 \%)$ & & \\
\hline IV & $73(20.6 \%)$ & & \\
\hline \multicolumn{4}{|l|}{$\begin{array}{l}\text { Tumor node } \\
\text { metastasis }\end{array}$} \\
\hline Yes & $108(30.4 \%)$ & & \\
\hline No & 247 (69.6\%) & & \\
\hline Distant metastasis & & - & - \\
\hline M0 & $332(93.5 \%)$ & - & - \\
\hline M1 & $23(6.5 \%)$ & - & - \\
\hline \multicolumn{4}{|l|}{ Histology } \\
\hline Papillary & $291(82.0 \%)$ & & \\
\hline Nonpapillary & $64(18.0 \%)$ & & \\
\hline
\end{tabular}

\section{Discussion}

In this study, we found that $+49 \mathrm{~A} / \mathrm{G}$ polymorphism elevated the risk of $\mathrm{BC}$ in Chinese Han population. Moreover, subgroup analyses suggested that the polymorphism was significantly correlated to age, smoking and drinking. This SNP was shown to have a link with tumor node metastasis and larger tumor size of $\mathrm{BC}$ patients.

Recently, Fang et al. designed a meta-analysis exploring the link between CTLA4+49A/G polymorphism and cancer susceptibility. They found that this polymorphism increased the risk of bone, liver, breast, pancreatic, and head and neck cancer; however, they found no association with the risk of gastric, colorectal, renal, or lung cancer [22], suggesting a potential cancer-specific effect. Wang et al. were the first research team to report a link between this SNP and BC susceptibility; their findings revealed that this polymorphism could decrease the risk of BC. After stratifying the BC 
TABLE 2: Logistic regression analysis of associations between CTLA- $4+49 \mathrm{~A} / \mathrm{G}$ polymorphism and risk of bladder cancer.

\begin{tabular}{|c|c|c|c|c|c|c|c|c|}
\hline \multirow{2}{*}{ Genotype } & \multicolumn{2}{|c|}{${ }^{\#}$ Cases $(n=355)$} & \multicolumn{2}{|c|}{${ }^{\#}$ Controls $(n=435)$} & \multirow{2}{*}{ OR $(95 \% \mathrm{CI})$} & \multirow{2}{*}{$P$} & \multirow{2}{*}{${ }^{*}$ OR $(95 \% \mathrm{CI})$} & \multirow{2}{*}{${ }^{*} P$} \\
\hline & $n$ & $\%$ & $n$ & $\%$ & & & & \\
\hline$\overline{\mathrm{AA}}$ & 206 & $58.2 \%$ & 288 & $66.3 \%$ & 1.00 & & 1.00 & \\
\hline AG & 127 & $35.9 \%$ & 132 & $30.5 \%$ & $1.35(0.99-1.82)$ & 0.055 & $1.35(1.00-1.82)$ & 0.054 \\
\hline GG & 21 & $5.9 \%$ & 14 & $3.2 \%$ & $2.10(1.04-4.22)$ & 0.038 & $2.10(1.04-4.23)$ & 0.038 \\
\hline $\mathrm{AG}+\mathrm{GG}$ & 148 & $41.8 \%$ & 146 & $33.7 \%$ & $1.42(1.06-1.89)$ & 0.019 & $1.42(1.06-1.90)$ & 0.018 \\
\hline $\mathrm{AA}+\mathrm{AG}$ & 333 & $94.1 \%$ & 420 & $96.8 \%$ & 1.00 & & 1.00 & \\
\hline GG & 21 & $5.9 \%$ & 14 & $3.2 \%$ & $1.89(0.95-3.78)$ & 0.071 & $1.90(0.95-3.79)$ & 0.070 \\
\hline A allele & 539 & $76.13 \%$ & 708 & $81.6 \%$ & 1.00 & & 1.00 & \\
\hline G allele & 169 & $23.87 \%$ & 160 & $18.4 \%$ & $1.39(1.09-1.77)$ & 0.008 & - & - \\
\hline
\end{tabular}

${ }^{\#}$ The genotyping was successful in 354 cases and 434 controls for CTLA $4+49 \mathrm{~A} / \mathrm{G}$; bold values are statistically significant $(P<0.05) .{ }^{*}$ Adjustments for age and sex.

TABLE 3: Stratified analyses between CTLA $-4+49 \mathrm{~A} / \mathrm{G}$ polymorphism and the risk of bladder cancer.

\begin{tabular}{|c|c|c|c|c|c|c|c|}
\hline \multirow{2}{*}{ Variable } & \multicolumn{3}{|c|}{ Case/control } & \multirow{2}{*}{$\begin{array}{c}\text { AG vs. AA } \\
\text { OR (95\% CI); } P\end{array}$} & \multirow{2}{*}{$\begin{array}{c}\text { GG vs. AA } \\
\text { OR (95\% CI); } P\end{array}$} & \multirow{2}{*}{$\begin{array}{l}\text { GG vs. AG + AA } \\
\text { OR }(95 \% \text { CI }) ; P\end{array}$} & \multirow{2}{*}{$\begin{array}{l}\text { GG + AG vs. AA } \\
\text { OR ( } 95 \% \text { CI); } P\end{array}$} \\
\hline & AA & AG & GG & & & & \\
\hline \multicolumn{8}{|l|}{ Sex } \\
\hline Male & $178 / 239$ & $107 / 111$ & $17 / 12$ & $1.29(0.93-1.80) ; 0.124$ & 1.90 (0.89-4.08); 0.099 & $1.74(0.82-3.70) ; 0.151$ & 1.35 (0.99-1.86); 0.060 \\
\hline Female & $28 / 49$ & $20 / 21$ & $4 / 2$ & 1.67 (0.77-3.60); 0.193 & 3.50 (0.60-20.34); 0.301 & $2.92(0.51-16.56) ; 0.404$ & 1.83 (0.87-3.82); 0.109 \\
\hline \multicolumn{8}{|l|}{ Smoking } \\
\hline Yes & $136 / 122$ & $99 / 49$ & $15 / 8$ & $1.81(1.19-2.76) ; 0.006$ & $1.68(0.69-4.11) ; 0.253$ & 1.36 (0.57-3.29); 0.489 & $1.79(1.20-2.68) ; 0.004$ \\
\hline No & $70 / 166$ & $28 / 83$ & $6 / 6$ & 0.80 (0.48-1.33); 0.392 & 2.37 (0.74-7.60); 0.147 & $2.54(0.80-8.07) ; 0.102$ & $0.91(0.56-1.47) ; 0.689$ \\
\hline \multicolumn{8}{|l|}{ Drinking } \\
\hline Yes & $130 / 110$ & $84 / 58$ & $18 / 5$ & 1.23 (0.81-1.87); 0.343 & 3.05 (1.10-8.47); 0.033 & $2.83(1.03-7.77) ; 0.044$ & $1.37(0.92-2.05) ; 0.127$ \\
\hline No & $76 / 178$ & $43 / 74$ & $3 / 9$ & 1.36 (0.86-2.16); 0.191 & 0.78 (0.21-2.96); 0.967 & $0.71(0.19-2.66) ; 0.839$ & 1.30 (0.83-2.03); 0.256 \\
\hline \multicolumn{8}{|l|}{ Age (years) } \\
\hline$<60$ & $87 / 126$ & $54 / 53$ & $13 / 9$ & $1.48(0.93-2.35) ; 0.103$ & 2.09 (0.86-5.11); 0.105 & 1.83 (0.76-4.41); 0.176 & 1.57 (1.01-2.43); 0.046 \\
\hline$\geq 60$ & $119 / 162$ & $73 / 79$ & $8 / 5$ & $1.26(0.85-1.87) ; 0.257$ & $2.18(0.70-6.83) ; 0.182$ & $2.01(0.65-6.24) ; 0.228$ & $1.31(0.89-1.93) ; 0.167$ \\
\hline
\end{tabular}

Bold values are statistically significant $(P<0.05)$.

TABLE 4: The associations between CTLA $4+49 \mathrm{~A} / \mathrm{G}$ polymorphism and clinical characteristics of bladder cancer.

\begin{tabular}{|c|c|c|c|c|}
\hline \multirow{2}{*}{ Characteristics } & \multicolumn{4}{|c|}{ Genotype distributions } \\
\hline & AA & AG & GG & $\mathrm{AG}+\mathrm{GG}$ \\
\hline \multicolumn{5}{|l|}{ Tumor grade } \\
\hline High/low & $139 / 67$ & $77 / 50$ & $11 / 10$ & $88 / 60$ \\
\hline OR (95\% CI); $P$ value & 1.00 (reference) & 0.74 (0.47-1.18); 0.204 & $0.53(0.22-1.31) ; 0.164$ & $0.71(0.46-1.10) ; 0.121$ \\
\hline \multicolumn{5}{|l|}{ Tumor size } \\
\hline$\geq 3 /<3$ & $42 / 164$ & $39 / 88$ & $11 / 10$ & $50 / 98$ \\
\hline OR (95\% CI); $P$ value & 1.00 (reference) & 1.73 (1.04-2.87); 0.033 & 4.30 (1.71-10.79); 0.001 & 1.99 (1.23-3.22); 0.005 \\
\hline \multicolumn{5}{|l|}{ TNM stage } \\
\hline $\mathrm{III}+\mathrm{IV} / \mathrm{I}+\mathrm{II}$ & $96 / 110$ & $70 / 57$ & $11 / 10$ & $81 / 67$ \\
\hline OR (95\% CI); $P$ value & 1.00 (reference) & $1.41(0.90-2.19) ; 0.131$ & $1.26(0.51-3.10) ; 0.613$ & 1.39 (0.90-2.12); 0.131 \\
\hline \multicolumn{5}{|l|}{ Tumor node metastasis } \\
\hline Yes/no & $55 / 151$ & $41 / 86$ & $12 / 9$ & $53 / 95$ \\
\hline OR (95\% CI); $P$ value & 1.00 (reference) & 1.31 (0.81-2.12); 0.274 & 3.66 (1.46-9.17); 0.004 & 1.53 (0.97-2.42); 0.066 \\
\hline \multicolumn{5}{|l|}{ Distant metastasis } \\
\hline $\mathrm{M} 1 / \mathrm{M} 0$ & $12 / 194$ & $8 / 119$ & $3 / 18$ & $11 / 137$ \\
\hline OR (95\% CI); $P$ value & 1.00 (reference) & 1.09 (0.43-2.74); 0.860 & 2.69 (0.70-10.44); 0.137 & 1.30 (0.56-3.03); 0.545 \\
\hline \multicolumn{5}{|l|}{ Histology } \\
\hline Papillary/nonpapillary & $166 / 40$ & $106 / 21$ & $19 / 2$ & $125 / 23$ \\
\hline OR (95\% CI); $P$ value & 1.00 (reference) & $1.22(0.68-2.18) ; 0.509$ & 2.29 (0.51-10.23); 0.414 & $1.31(0.75-2.30) ; 0.347$ \\
\hline
\end{tabular}

Bold values are statistically significant $(P<0.05)$. 
patients, the associations between the polymorphism and clinical parameters including grade, stage, and histological types were not significant, suggesting that this SNP likely does not contribute to $\mathrm{BC}$ development and progression [19]. A subsequent study, however, indicated the presence of $+49 \mathrm{~A} / \mathrm{G}$ polymorphism was related to increased susceptibility to BC and that this SNP increased the susceptibility of BC among smokers. However, CTLA- $4+49 \mathrm{~A} / \mathrm{G}$ had no association with tumor stage or grade in BC patients [20]. Our findings suggested that this SNP elevated the risk of BC. The fact that these studies involved $\mathrm{BC}$ patients differing in ethnicity (and thus having distinct genetic backgrounds) might explain the discrepant findings. Differences in sample size, clinical heterogeneity of the cohorts, and diversity in the environmental exposure factors and lifestyle among geographical regions might also contribute to the conflicting results.

We obtained a significant relationship between the $+49 \mathrm{~A} / \mathrm{G}$ polymorphism and BC susceptibility among smokers and drinkers, suggesting that the presence of this SNP in combination with smoking/drinking may further increase the risk of BC. Furthermore, this SNP was linked with tumor node metastasis and tumor size of BC. We found no relationship between this polymorphism and TNM stage or tumor grade, in line with previous findings [20].

This study had several limitations. First, the findings of this study could not be extrapolated to other races with different genetic backgrounds. Second, the cohort size was small, especially after stratification of the patients. Third, we focused on a SNP in the CTLA-4 gene, thus ignoring the impact of potential interactions among polymorphisms. Fourth, the impact of $+49 \mathrm{~A} / \mathrm{G}$ polymorphism on the transcription and translation of CTLA- 4 gene was not investigated. Fifth, data on certain environmental factors that $\mathrm{BC}$ patients are exposed to were not available for further analysis.

To sum up, this study observes that CTLA- $4+49 \mathrm{~A} / \mathrm{G}$ polymorphism increases the risk of $\mathrm{BC}$ in Chinese individuals. The findings of this study require validation in future large cohort studies.

\section{Data Availability}

The data of this study can be obtained from the corresponding author upon request.

\section{Conflicts of Interest}

The authors declare that they have no conflict of interest.

\section{References}

[1] J. Ferlay, M. Colombet, I. Soerjomataram et al., "Estimating the global cancer incidence and mortality in 2018: GLOBOCAN sources and methods," International Journal of Cancer, vol. 144, no. 8, pp. 1941-1953, 2019.

[2] S. Antoni, J. Ferlay, I. Soerjomataram, A. Znaor, A. Jemal, and F. Bray, "Bladder cancer incidence and mortality: a global overview and recent trends," European Urology, vol. 71, no. 1, pp. 96-108, 2017.
[3] M. Moschini, D. D'Andrea, S. Korn et al., "Characteristics and clinical significance of histological variants of bladder cancer," Nature Reviews Urology, vol. 14, no. 11, pp. 651-668, 2017.

[4] R. Zigeuner, "Bladder cancer in 2016: news in diagnosis, treatment, and risk group assessment," Nature Reviews Urology, vol. 14, no. 2, pp. 74-76, 2017.

[5] S. K. Bhanvadia, "Bladder cancer survivorship," Current Urology Reports, vol. 19, no. 12, p. 111, 2018.

[6] M. A. Knowles and C. D. Hurst, "Molecular biology of bladder cancer: new insights into pathogenesis and clinical diversity," Nature Reviews: Cancer, vol. 15, no. 1, pp. 25-41, 2015.

[7] M. I. Fernández, M. Brausi, P. E. Clark et al., "Epidemiology, prevention, screening, diagnosis, and evaluation: update of the ICUD-SIU joint consultation on bladder cancer," World Journal of Urology, vol. 37, no. 1, pp. 3-13, 2019.

[8] N. Rothman, M. Garcia-Closas, N. Chatterjee et al., "A multistage genome-wide association study of bladder cancer identifies multiple susceptibility loci," Nature Genetics, vol. 42, no. 11, pp. 978-984, 2010.

[9] R. Kandimalla, A. A. G. van Tilborg, L. C. Kompier et al., "Genome-wide analysis of CpG island methylation in bladder cancer identified TBX2, TBX3, GATA2, and ZIC4 as pTaspecific prognostic markers," European Urology, vol. 61, no. 6, pp. 1245-1256, 2012.

[10] M. Wang, Z. Li, H. Chu et al., "Genome-wide association study of bladder cancer in a Chinese cohort reveals a new susceptibility locus at 5q12. 3," Cancer Research, vol. 76, no. 11, pp. 32773284, 2016.

[11] E. Valk, C. E. Rudd, and H. Schneider, "CTLA-4 trafficking and surface expression," Trends in Immunology, vol. 29, no. 6, pp. 272-279, 2008.

[12] C. B. Thompson and J. P. Allison, "The emerging role of CTLA-4 as an immune attenuator," Immunity, vol. 7, no. 4, pp. 445-450, 1997.

[13] W. Zhang, L. Shi, Z. Zhao et al., "Disruption of CTLA-4 expression on peripheral blood CD8 + T cell enhances antitumor efficacy in bladder cancer," Cancer Chemotherapy and Pharmacology, vol. 83, no. 5, pp. 911-920, 2019.

[14] L. van Hooren, L. C. Sandin, I. Moskalev et al., "Local checkpoint inhibition of CTLA-4 as a monotherapy or in combination with anti-PD1 prevents the growth of murine bladder cancer," European Journal of Immunology, vol. 47, no. 2, pp. 385-393, 2017.

[15] N. N. Davarpanah, A. Yuno, J. B. Trepel, and A. B. Apolo, "Immunotherapy," Current Opinion in Oncology, vol. 29, no. 3, pp. 184-195, 2017.

[16] C. Wang, W. Liu, L. Zhao, and Z. Dong, "Association of cytotoxic T-lymphocyte antigen-4 + 49A/G gene polymorphism with hepatocellular carcinoma risk in Chinese," Journal of Cancer Research and Therapeutics, vol. 14, pp. S1117-S1120, 2018.

[17] L. He, T. Deng, and H. S. Luo, “Association between cytotoxic T-lymphocyte antigen- $4+49 \mathrm{~A} / \mathrm{G}$ polymorphism and colorectal cancer risk: a meta-analysis," International Journal of Clinical and Experimental Medicine, vol. 8, no. 3, pp. 3752-3760, 2015.

[18] N. Bilbao-Aldaiturriaga, A. Patino-Garcia, I. Martin-Guerrero, and A. Garcia-Orad, "Cytotoxic T lymphocyte-associated antigen 4 rs231775 polymorphism and osteosarcoma," Neoplasma, vol. 64, no. 2, pp. 299-304, 2017.

[19] L. Wang, G. Su, X. Zhao et al., "Association between the cytotoxic T-lymphocyte antigen $4+49 \mathrm{~A} / \mathrm{G}$ polymorphism and 
bladder cancer risk," Tumour Biology, vol. 35, no. 2, pp. 11391142, 2014.

[20] P. K. Jaiswal, V. Singh, and R. D. Mittal, "Cytotoxic T lymphocyte antigen 4 (CTLA4) gene polymorphism with bladder cancer risk in North Indian population," Molecular Biology Reports, vol. 41, no. 2, pp. 799-807, 2014.

[21] F. Mao, X. B. Niu, S. Gu, L. Ji, B. J. Wei, and H. B. Wang, “The association between matrix metalloproteinase- 7 genetic variant and bladder cancer risk in a Chinese Han population," Clinical and Experimental Medicine, vol. 19, no. 4, pp. 565570, 2019.

[22] M. Fang, W. Huang, D. Mo, W. Zhao, and R. Huang, "Association of five SNPs in cytotoxic T-lymphocyte antigen 4 and cancer susceptibility: evidence from 67 studies," Cellular Physiology and Biochemistry, vol. 47, no. 1, pp. 414-427, 2018. 